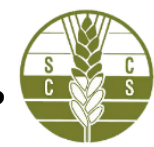

\title{
RESPUESTA DEL CILANTRO (Coriandrum sativum L.) A DISTINTAS PROPORCIONES DE COMPOST EN CONDICIONES SEMICONTROLADAS EN CORDOBA - COLOMBIA.
}

\author{
Eliecer M. Cabrales $H^{1}$. $\$ y Jesús A. Ayala $C^{2}$
}

1 PhD. Universidad de Córdoba. Grupo de investigación Agricultura sostenible.

ecabralesh@correo.unicord oba.edu.co;

ecabralesh@yahoo.es

2 Ingeniero Agrónomo, Grupo de investigación Agricultura sostenible Universidad de Córdoba.

\section{Palabras Claves:}

Cilantro, Compost y producción, abono orgánico, cilantro fresco

\section{RESUMEN}

En aras de contribuir en la seguridad alimentaria y en especial en la producción de hortalizas en clima cálido, se planteó esta investigación en la que se evaluó la respuesta de un abono orgánico compostado localmente en el desarrollo de plantas de cilantro en condiciones semicontroladas en clima cálido en el departamento de Córdoba. Esta investigación se fundamenta en que es un cultivo que requiere de abundante materia orgánica y los suelos agrícolas del departamento de Córdoba, suelen tener bajos tenores. Se utilizó un diseño experimental completo al azar, con cinco tratamientos (0, 25, 50, 75 y 100\% de abono orgánico) y tres repeticiones. Cada unidad experimental estuvo conformada por nueve materos en las que se sembró el cilantro a $15 \mathrm{~cm}$ entre plantas. Se evaluó número y longitud de hojas, y los rendimientos. La información se almacenó en tablas de Excel y se hizo análisis de varianza y las pruebas de comparación de medias de Tukey con un nivel de confianza del 95\%, para lo cual se utilizó se el programa estadístico S.A.S versión 9.4. La mejor respuesta en el ensayo se logró con el 50\% de abono orgánico de producción local, obteniéndose el mayor número de hojas y rendimiento. Se concluye que para la producción de cilantro en condiciones de clima cálido y en suelos agrícolas, se requiere de aplicaciones de abono orgánico, proporciones del $50 \%$ son suficientes para lograr los mejores rendimientos del cultivo.

\section{RESPONSE OF CORIANDER (Coriandrum sativum L.) TO VARIOUS PROPORTIONS OF COMPOST IN SEMICONTROLED CONDITIONS IN CORDOBA - COLOMBIA.}

1 PhD. Universidad de Córdoba. Grupo de investigación Agricultura sostenible.

ecabralesh@correo.unicord oba.edu.co

ecabralesh@yahoo.es

2 Ingeniero Agrónomo, Grupo de investigación Agricultura sostenible Universidad de Córdoba. jayala@fca.edu.co

\section{Palabras Claves:}

Coriander, compost and production, organic fertilizer, fresh coriander.

\section{RESUMEN}

In order to contribute to food security and especially in the production of vegetables in warm climate, this research was proposed in which the response of a locally composted organic fertilizer in the development of coriander plants in semi-controlled conditions in warm climate was evaluated in the department of Córdoba. This research is based on the fact that it is a crop that requires abundant organic matter and the agricultural soils of the department of Córdoba tend to have low contends. A complete randomized experimental design was used, with five treatments ( 0 , 25, 50, 75 and 100\% organic fertilizer) and three replications. Each experimental unit constituted of nine pots in which coriander was sown to $15 \times 11 \mathrm{~cm}$ between plants. Were evaluated: number and length of leaves, and yields. The information was stored in Excel tables and did was analysis of variance and Tukey's comparison tests of means were made with a confidence level of 95\%, for which the statistical program S.A.S version 9.4 was used. The best response was achieved with $50 \%$ locally produced organic fertilizer, obtaining the highest number of leaves and yield. It is concluded that for the production of coriander in hot climate conditions and in agricultural soils, organic fertilizer applications are required, proportions of $50 \%$ are sufficient to achieve the best this crop yields 


\section{INTRODUCCIÓN}

Según el MADR (2016), Colombia tiene una producción hortícola de 1,6 millones de toneladas que se encuentran dispuestas en poco más de 100.000 hectáreas en todo el país. Teniendo en cuenta la distribución espacial de estos cultivos, el DANE (2016) presento que en el departamento de Córdoba se encuentran sembradas 4.909 hectáreas en producción de hortalizas de las 1.833.931 ha con vocación agrícola. El cultivo de cilantro en nuestro país se logra cultivar principalmente en el departamento de Cundinamarca con un área establecida de 1.963 hectáreas de las 3.000 existentes en esa región. Los rendimientos del cilantro en Colombia en condiciones óptimas, oscilan entre 18 y 20 toneladas por hectárea de follaje verde, la cual se logra con una población de 200 a 300 plantas por metro cuadrado (Arcos et al., 2002; Estrada, 2000; Mejía et al., 2008).

El cilantro es una planta de la familia Apiacea, a la que pertenecen varios grupos de plantas medicinales, aromáticas y de condimento de mayor consumo, es una planta con un rápido y fácil crecimiento, se adapta a características de pleno sol, al igual que en sombrío parcial (Marangoni et al., 2011; Rajeshwari y Andallu, 2011; Ramadan et al., 2003; Valdiviezo, 2011). Díaz (2017) a su vez manifiesta que la planta de cilantro se caracteriza por poseer un sistema radicular axonomorfo, muy delgado y altamente ramificado, posee tallo dicotómico, herbáceo, hueco y erecto que en algunos casos presenta una serie de brotes laterales, hojas inferiores pinnadas hasta tripinnadas con segmentos ovales en forma silifirma, inflorescencia en umbela compuesta y frutos ovalados y globulares que se pueden identificar por ser diaquenios globosos. Masabni y Lillard (2013), manifiestan que el cultivo de cilantro tiene una adaptación a climas cálidos, frescos y fríos, sin embargo, su mejor desarrollo se logra en el clima templado, donde se logran los mayores rendimientos. La temperatura óptima oscila entre los 15 y $20^{\circ} \mathrm{C}$ (Morales et al., 2015), con una precipitación pluvial de $350 \mathrm{~mm}$, humedad relativa del $75 \%$, intensidad lumínica de 5-6 horas por día, altitud hasta $2.200 \mathrm{msnm}$ y suelo franco arenoso a franco arcilloso, bien drenados, cuyo $\mathrm{pH}$ oscile $6.5-7.5$ y que contengas buenos contenidos de materia orgánica.

Como fuente de materia orgánica están los abonos compostados, cuya elaboración se hace con materiales orgánicos de producción local (desechos de cortes de césped, ramas, hojarascas, desechos de hogares, entre otros). El compost por su riqueza mineral, puede sustituir parcialmente los fertilizantes de síntesis y considerársele como una alternativa de manejo de suelos dedicados a monocultivos, que son altamente dependientes de fertilizantes de origen químico, los que, a su vez, incrementa los costos de producción (Campos et al., 2020). El uso continuo de fertilizantes químicos conlleva a impactos negativos en la diversidad rizosférica del suelo, afectando de forma directa los procesos biológicos naturales y la productividad agrícola (Cruz, Alayón y Morón, 2017). La fertilización química por su alta solubilidad, proporciona nutrientes que rápidamente son aprovechables por las plantas, sin embargo, al mal uso y excesivo, puede generar problemas de acidez o de salinidad y con ello el deterioro de la calidad y salud del suelo (Aguiñaga et al. 2020).

La aplicación de abono orgánico al suelo contribuye con la agregación de las partículas minerales y con ello, se mejora la estructura, aireación, penetración de raíces, retención de humedad, disminuye la erosión y facilita el laboreo. Adicional a ello, el tamaño coloidal y la carga negativa, retienen de forma intercambiable los cationes 
esenciales. Así mismo, el material orgánico actúa como agente amortiguador al impedir los cambios bruscos del pH (Flores et al., 2016). En este orden de ideas la agricultura moderna incorpora la utilización de productos orgánicos en los sistemas de producción incrementando el crecimiento y el rendimiento de los cultivos, calidad de las cosechas y el efecto que tienen sobre el alargamiento celular, la diferenciación vascular y el desarrollo de las plantas (Aguiñaga et al., 2020).Estrada (2010) indica que los abonos orgánicos en los sistemas de producción hortícola, juegan un papel importante en la mitigación del calentamiento global, es amigable con el medio ambiente y contribuye a la sostenibilidad de la agricultura. En este sentido, la FAO (2013) señala que la descomposición de estos materiales orgánicos se genera en presencia de oxígeno (aeróbica), humedad y temperatura regulada, condiciones ideales para el desarrollo de micro y macro-organismos, quienes se encargan de transformar este material orgánico, en uno más estable.

Con base en esta revisión, se planteó esta investigación en la que se evaluó el efecto de varias dosis de compost de producción local en el cultivo de cilantro en condiciones semicontroladas en el departamento de Córdoba-Colombia. Con la cual se busca incentivar la producción de esta hortaliza en condiciones locales, promoviendo la mano obra y mejoramiento de la calidad de vida de los pequeños productores de este departamento.

\section{MATERIALES Y MÉTODOS}

Ubicación. Esta investigación se realizó en condiciones semicontroladas en los lotes experimentales de la Universidad de Córdoba, zona cálida del caribe colombiano, en el Valle del Sinú Medio, situado a $80^{\circ} 47^{\prime}$ LN y $75^{\circ} 51^{\prime}$ LW con respecto al meridiano de Greenwich, altura de 15 m.s.n.m. La zona ecológica corresponde al bosque seco tropical (BS-T) con temperatura promedio de $28^{\circ} \mathrm{C}$, humedad relativa media del $83 \%$ y precipitación anual de $1200 \mathrm{~mm}$ (Palencia et al., 2006).

Preparación del sustrato y siembra. Inicialmente los sustratos (suelo aluvial del río Sinú y abono orgánico compostado localmente) se sometieron a desinfección por el método de solarización, la cual se hizo en capas de $10 \mathrm{~cm}$ aproximadamente y recubiertas por plástico de color negro, por 3 días y expuestas al sol, posteriormente se dejó en reposo por 3 días. Seguidamente se tomaron muestras para el análisis químico de los mismos (IGAC, 2006). Con los sustratos desinfectados, se prepararon las relaciones suelo: abono orgánico $(0,25,50,75$ y $100 \%$ de abono orgánico), cada mezcla fue vertida en materos de 30 litros de capacidad, el reacomodo de partículas se hizo con riego (0,5 litros de agua cada 15 minutos) hasta saturación total y se dejó drenar por 2 días. La siembra se hizo con semilla comercial oro verde, depositando cuatro semillas por sitio a una distancia $15 \times 11 \mathrm{~cm}$ entre planta.

Manejo del ensayo. El ensayo se estableció con polisombra al $50 \%$ de sombra, se aplicaba riego frecuente según necesidad de cada unidad experimental. El manejo de arvenses en el ensayo se realizó de forma manual en el momento que estas se iban presentando, no se presentaron ataques de insectos. Se trabajó con la oferta nutricional de cada sustrato, es decir, no se hizo planes de fertilización, no se presentaron deficiencias nutricionales visibles durante el ensayo.

Diseño experimental. Se trabajó con un diseño experimental completo al azar evaluando 5 tratamientos $(0,25,50,75$ y $100 \%$ de abono orgánico) y 3 repeticiones. Cada unidad experimental estuvo 
conformada por nueve materos en las que se sembró el cilantro a $15 \mathrm{~cm}$ entre plantas. Cada matero tenía $30 \times 40 \mathrm{~cm}$ y $25 \mathrm{~cm}$ de profundidad.

Evaluación de variables. Se evaluaron las variables de crecimiento y desarrollo de mayor importancia en este cultivo: número de hojas (unidades por planta), longitud de la hoja (centímetros) y rendimiento (kilogramos por hectárea) al igual que la absorción de nutrientes por parte de las plantas de cilantro.

Análisis de la información. Todos los datos obtenidos se tabularon en tablas de Excel y se procesaron con el programa estadístico S.A.S versión 9.4 realizando análisis de varianzas, y las pruebas comparativas de media por Tukey $(\mathrm{p} \leq 5 \%)$.

\section{RESULTADOS Y DISCUSIÓN}

Caracterización de sustratos. El análisis fisicoquímico del suelo aluvión de textura franca, indica que es de reacción ácida, bajo contenido de materia orgánica, por lo tanto, baja oferta de nitrógeno para las plantas; alto contenido de azufre, pero no excesivo, cantidad que no es suficiente para bajar el $\mathrm{pH}$ del suelo. Aunque el contenido de fósforo es medio, este podría ser deficitario, ya que se podría precipitar junto con calcio que es excesivo. Los contenidos de bases intercambiables son altos, para el calcio y el magnesio, guardan buena relación, potasio está en contenidos bueno y no hay problemas de sodio, como tampoco de aluminio. En términos generales este suelo aluvial no tiene problemas químicos, es de mediana oferta nutricional. Por otro lado, el abono orgánico compostado localmente, se logró en 60 días para lo cual se utilizó desechos sólidos de la Universidad de Córdoba, este material finalmente quedó con un $\mathrm{pH}$ cercano a la neutralidad y con contenido medio de materia orgánica oxidable, ofrece mayores contenidos de azufre, fósforo, y bases intercambiables, pero sin problemas de sodio, como tampoco de aluminio (Tabla $1)$.

Tabla 1. Resultados de los análisis realizados a los sustratos del ensayo: suelo aluvial del río Sinú y Compost de producción local.

\begin{tabular}{ccccccccccc}
\hline \multirow{2}{*}{ Sustrato } & $\mathbf{p H}$ & $\mathbf{M . O}$ & $\mathbf{S}$ & $\mathbf{P}$ & $\mathbf{C a}$ & $\mathbf{M g}$ & $\mathbf{K}$ & $\mathbf{N a}$ & $\mathbf{A l + H}$ & $\mathbf{C I C e}$ \\
\cline { 2 - 11 } & $1: 1$ & \multicolumn{2}{c}{$\%$} & \multicolumn{2}{c}{$\mathrm{mg} / \mathrm{kg}$} & \multicolumn{1}{c}{$\mathrm{cmol}^{(+)} / \mathrm{kg}$} & & \\
\hline Suelo aluvial & 5,44 & 1,07 & 46,8 & 17,4 & 12,4 & 6,3 & 0,3 & 0,08 & 0,2 & 19,3 \\
\hline Compost local & 6,74 & 2,97 & 270,6 & 259,7 & 21,2 & 8 & 6,27 & 0,7 & 0 & 36,2 \\
\hline
\end{tabular}

Número de hojas. Esta variable osciló entre 8,5 y 11,8 unidades/planta, con diferencias altamente significativas $(\mathrm{p}<0.001)$ entre aplicación y sin aplicación de abono orgánico, pero entre los tratamientos con abono orgánico, no hubo diferencias (Tabla 2). El Tratamiento con el $50 \%$ de abono orgánico, fue el de mayor producción de hojas, con 11,8 unidades/planta, seguida por los tratamientos 75 y $25 \%$ con 11,4 hojas respectivamente, un bajo coeficiente de variación (5,98\%) (Figura 1). Estos resultados son similares a los reportados por Hernández (2004), quien encontró 
diferencias estadísticas significativas al evaluar el efecto de abonos orgánicos en la producción de cilantro, manifestando que la renovación de follaje en las plantas de cilantro, está arraigada a la cantidad de materia orgánica y nutrientes que contiene cada sustrato, ya que esta es determinante en los aportes nutrimentales y capacidad de retención de humedad. De igual forma Salgado et al., (2020), indican que el uso de abonos orgánicos en la producción de cilantro, contribuye a mejorar las características de la planta con fines antioxidantes, toda vez que el material orgánico en el suelo ejerce una acción física que favorece la agregación del mismo, al igual como medio alimenticio, se mejoran los aportes biológicos como sustancias cementantes, aglutinantes, químicas y bioquímicas que conllevan a un mejor desarrollo del cultivar.

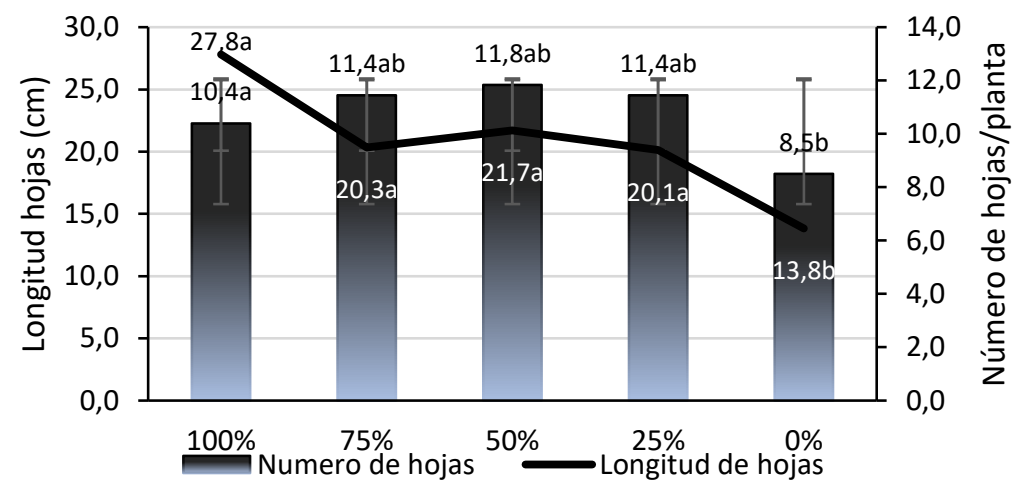

Figura 1. Número y longitud de hojas del cilantro con varias dosis de compost de producción local.

Tabla 2. Cuadrados medios de número de hojas (NH), longitud de hojas ( $\mathrm{LH})$, y rendimiento (RTO) del cilantro en condiciones de clima cálido.

\begin{tabular}{ccccc}
\hline Fuente de Variación & GL & NH & LH & RTO \\
\hline Tratamientos & 4 & $5.49537^{* *}$ & $74.4103^{* *}$ & $1.086 \mathrm{E}+08^{* *}$ \\
Error & 10 & 0.41111 & 10.7810 & 5584157 \\
Coeficiente de variación (\%) & & 5.98 & 15.81 & 13.64
\end{tabular}

** Diferencia estadística significativa al $1 \%$ de probabilidad. NH: numero de hojas. LH: longitude de hoja. RTO: rendimiento.

Longitud de la hoja. Las hojas del cilantro tuvieron un crecimiento que osciló entre 13,8 y $27,8 \mathrm{~cm}$, con diferencias altamente significativas $(\mathrm{p}<0.001)$ entre los tratamientos con y sin aplicación del compost (Tabla 2), lo que deja entrever la importancia en el desarrollo del cultivar. En esta longitud se incluye el peciolo de la hoja. La prueba de comparación de medias de Tukey indica el tratamiento con la hoja corta 
se obtuvo cuando no se aplicó compost al suelo, y la mayor longitud se logra con el $100 \%$ de compost, sin embargo, entre los tratamientos abonados con el compost, no hubo diferencias estadísticas significativas (Figura 1). Estos resultados contrastan a los reportados por Torres (2012) al no encontrar diferencias significativas en la evaluación de abonos, dosis y la interacción de estos en una hortaliza similar (apio). El uso de compost ofrece ventajas en términos de operación, incremento del carbono, retención de agua, aportes de minerales, sin embargo, pueden existir desventajas, entre ellas: incremento de sales en el sustrato, toxicidad por algunos elementos contaminantes del compost (metales pesados).
Rendimiento. Los rendimientos de este cultivar oscilaron entre 6629 y $20868 \mathrm{~kg} / \mathrm{ha}$ de la parte aérea, con diferencias estadísticas altamente significativas (Tabla 2) entre los tratamientos con y sin aplicación de compost ( $\mathrm{p}<0.001)$. Por comparación de medias (Tukey), se pudo observar que no hay diferencias entre los tratamientos que recibieron aplicación de compost, sin embargo, en la figura 2 se puede apreciar que al aplicar el 50\% de compost, se logra el máximo rendimiento del cultivar., pero si no se aplica, este rendimiento es de $6629 \mathrm{~kg} / \mathrm{ha}$, esto muestra la importancia de la aplicación del compost, material que se puede producir con los residuos sólidos de las fincas.

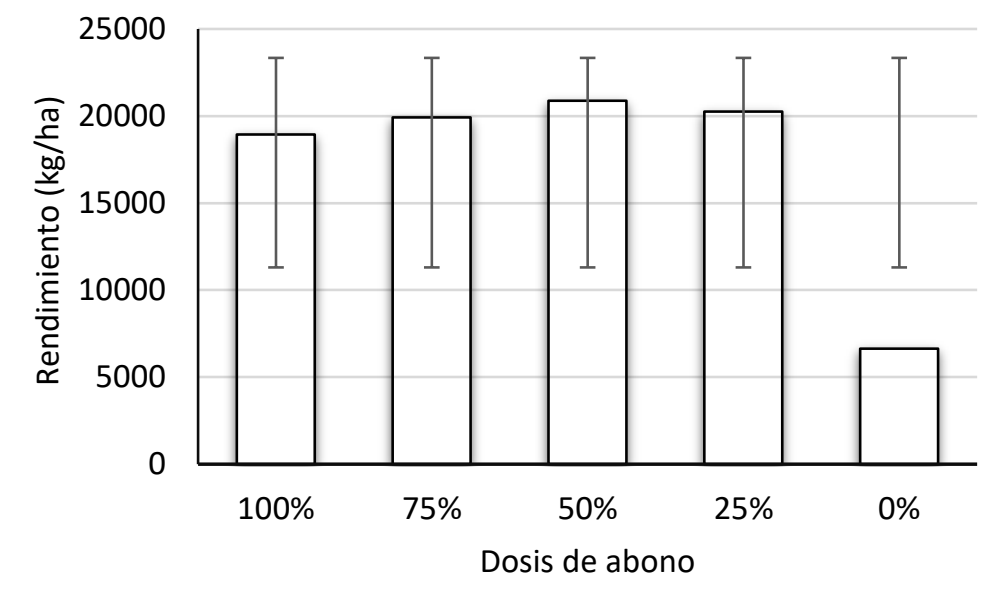

Figura 2. Rendimiento del cultivo de cilantro con distintas dosis de compost de producción local.

Estos resultados son similares a los que reporta Ozuna (2015), quien encontró diferencias estadísticas altamente significativas al evaluar el efecto de fertilizantes órgano-minerales en el rendimiento y calidad del cultivo de cilantro, quien manifiesta que este tipo de fertilizante promueve una mejor absorción de nutrientes, recuperación de la fertilidad del suelo, mejoramiento estructural y retención de humedad, entre otros en el mismo.
De no aplicarse compost, no se superan los $7256 \mathrm{~kg} / \mathrm{ha}$ que reporta Tituaña et al., (2019) en suelos agrícolas ecuatorianos, por lo que es importante agregar material orgánico al suelo para este tipo de cultivo, por todas las bondades que esto trae consigo. Así mismo, estos mismos autores reportan rendimientos de hasta $9847 \mathrm{~kg} / \mathrm{ha}$ en este cultivar, cuando utilizaron 25 y $50 \%$ de material de sedimento rico en material orgánico, rendimiento superado por este ensayo, posiblemente por los aportes nutricionales 
del compost al suelo, toda vez que es un material estable, rico en microorganismos que contribuyen de manera directa $\mathrm{e}$ indirecta en la nutrición de las plantas.

Absorción de nutrientes. Esta variable se vio favorecida por la cantidad de compost agregado al suelo (Tabla 3), sin embargo, no superó los 0,54\% que reporta Imankulova et al., (2020) en análisis foliares de esta planta, pero son similares las concentraciones de magnesio y fósforo, quienes reportan $0,04 \%$ para ambos elementos. En cuanto al calcio, los valores obtenidos en esta investigación superan grandemente a los $0,06 \%$ reportados por estos mismos autores. Para el caso del nitrógeno, los valores obtenidos están en el rango de 2,7 - 3,9\% que reportan Cruz et al., (2017), sin embargo, cuando no se aplica compost, este valor es de $2,1 \%$, es decir, que el compost contribuye al enriquecimiento de nitrógeno del cilantro. Estos resultados proporcionan información importante sobre la riqueza mineral del follaje de esta planta, logrado solo con los aportes del compost.

Tabla 3. Resultados de los análisis foliares para las muestras evaluadas en el laboratorio.

\begin{tabular}{cccccccc}
\hline $\begin{array}{c}\text { Dosis Compost } \\
(\%)\end{array}$ & $\mathbf{N a}$ & $\mathbf{S}$ & $\mathbf{M g}$ & $\mathbf{P}$ & $\mathbf{C a}$ & $\mathbf{N}$ & $\mathbf{K}$ \\
\cline { 2 - 7 } & & & & $\mathbf{\%}$ & & & \\
\hline $\mathbf{1 0 0}$ & 0,16 & 0,16 & 0,33 & 0,51 & 1,05 & 2,98 & 8,06 \\
\hline $\mathbf{7 5}$ & 0,05 & 0,14 & 0,35 & 0,72 & 0,89 & 2,89 & 8,05 \\
\hline $\mathbf{5 0}$ & 0,08 & 0,12 & 0,31 & 0,58 & 0,89 & 3,28 & 7,79 \\
\hline $\mathbf{2 5}$ & 0,07 & 0,10 & 0,32 & 0,46 & 1,13 & 3,52 & 6,64 \\
\hline $\mathbf{0}$ & 0,06 & 0,12 & 0,46 & 0,46 & 1,25 & 2,11 & 6,23 \\
\hline
\end{tabular}

La importancia de tener plantas con suficiencias de minerales radica en que estos serán aprovechados por el cuerpo humano al> consumirlas, en este sentido, Leal (2018) reporta que el cuerpo humano requiere de minerales como el calcio (184 mg), fósforo (71 mg), hierro (1,42 mg), entre otros, los cuales son fácilmente aprovechables de los vegetales como el cilantro que es de> consumo fresco, y la riqueza nutricional de una planta está dada por la disponibilidad que haya en el sustrato donde se produce. Morales et al. 2013, a su vez manifiesta que el contenido excesivo de nitrógeno en las plantas, la predispone al ataque de plagas y enfermedades.

\section{CONCLUSIONES}

En condiciones edafoclimáticas de Córdoba, es posible producir cilantro, siempre y cuando se le adicione al suelo material compostado en dosis del 25 al 50\%, con lo cual no es necesario aplicar planes de fertilización sintética.

Con el uso de compost, se mejoran los componentes fisiológico número y longitud de hojas, los cuales redundan de manera positiva en el rendimiento del cilantro en condiciones agroclimáticas de Córdoba.

\section{REFERENCIAS}

Aguiñaga, A., Medina K., Garruña, R., Latournerie, L. y Ruíz, E. (2020). Efecto de abonos orgánicos sobre el rendimiento, valor 
nutritivo y capacidad antioxidante de tomate verde (Physalis ixocarpa). Acta Universitaria 30, e2475.

Arcos, L.; Estrada, I. y Muñoz, J. (2002). Estabilidad de cinco cultivares de cilantro (Coriandrum sativum L.) en cinco niveles de nitrógeno y dos épocas de siembra. Trabajo de grado. Ing. Agrónomo. Palmira. Universidad Nacional de Colombia.

Campos, J., Álvarez, M., Maldonado, R. y Almaguer, G. (2020). Aplicación de abonos orgánicos en el rendimiento y desarrollo radicular en el cultivo de aguacate. Revista Mexicana de Ciencias Agrícolas 11(2):1-12.

Cruz, E., Can, A., Loera, L., Aguilar, G., Pineda, J., Bugarín, R. (2017). Extracción de N-P-K en Coriandrum sativum 'Pakistan' en hidroponía. Revista Mexicana Ciencias Agrícolas, 8(2): 355367.

Cruz, P., Alayón, A. y Morón, A. (2017). Efecto de la fertilización orgánica y de síntesis química en tomate verde (Physalis ixocarpa Brot. Ex Horn) en Calakmul Campeche(México). Avances en Investigación Agropecuaria, 21(2), 41-53. DANE, (2016). Encuesta nacional agropecuarias. On line: https://www.dane.gov.co/files/investigacion es/agropecuario/enda/ena/2015/boletin_ena 2015.pdf.

Díaz, Y. (2017). Cultivo de cilantro (Coriandrum sativum L.) para obtención de semillas. Academia, San Francisco. 1p.

Estrada, A. (2010). Manual elaboración de abonos orgánicos sólidos, tipo compost. ICTA-CIAL. Guatemala. 1-2.

Estrada, I. (2000). El cultivo de cilantro UNAPAL Precoso. Programa de Investigación en Hortalizas. Trabajo de grado Ingeniero Agrónomo. Palmira. Universidad Nacional de Colombia. 23p.

FAO, (2013). Organic Agriculture. On line: http://termportal.fao.org/faooa/main/start.do . [Agosto de 2017].

Flores, P., Lima, J., Santana, J., Reino, L., Beja, P. and Moreira, F. (2016). An applied farming systems approach to conservationrelevant agricultural practices for agrienvironment policy design. Land Use Policy. 58(1):165-172.

Hernández, Arturo. (2004). Efecto de dos abonos orgánicos en la producción de cilantro (Coriandrum sativum L.) bajo condiciones de campo en Villaldama Nuevo León. Trabajo de grado Ingeniero Agrónomo. Universidad Autónoma Antonio Narro. Buenavista, Saltillo, Coahuila, México. Pág. 29.

Imankulova, G., Moldabayeva, Z., Mammadov, R., Kassenov, A., Utegenova, A. (2020). Nutritional value and antioxidant activity of brassica oleracea and coriandrum sativum vegetable crops. EurAsian Journal of BioSciences, 14: 901-906.

Instituto Geográfico Agustín Codazzi. (IGAC) (2006). Informe de Gestión 2006. On line. http://www.igac.gov.co:10040/wps/wcm/conne ct/61a76300428cb3a699befd6bc8a4ca26/Infor me+de+Gestion_2006+copia.pdf?MOD=AJPE RES. [enero - diciembre, 2006].

Leal, K. (2018). Mejoramiento genético para la obtención de nuevas poblaciones de cilantro (Coriandrum sativum L.). Tesis de Maestría Ciencias Agrarias. Universidad Nacional de Colombia - Sede Palmira. p16.

Marangoni, C. y Fernández de Moura, N. (2011). Antioxidant activity of essential oil from Coriandrum sativum L. in Italian salami. Ciencia y Tecnología de Alimentos 31(1): 124-128.

Masabni, J. y Lillard, P. (2013). Jardinería fácil. Texas A\&M Agri-Life. Texas EE.UU. $1-3 p$. 
Mejía, S.; Estrada, E. y Figueroa, O. (2008). Respuesta fisiológica del cilantro a diferentes niveles de potasio y nitrógeno. Acta agronómica. Universidad Nacional de Colombia sede Palmira. 57(3): 4.

Ministerio de Agricultura y Desarrollo Rural (MADR), (2016). Informe rendición de cuentas del ministerio de agricultura y desarrollo rural. On line: https://www.minagricultura.gov.co/Documento s\%20Publicos/RENDICION\%20DE\%20CUEN TAS\%20-\%20INFORME\%2020162017.pdf\#search=cilantro.

Ministerio de Agricultura y Desarrollo Rural. (MADR). (2009). Anuario estadístico de frutas y hortalizas 2004- 2008. Bogotá. 214-216.

Morales J., Brunner B., Flores L. y Martínez S. (2013). Proyecto de Agricultura Orgánica. Hoja informativa. Departamento de Cultivos y Ciencias Agroambientales. Estados Unidos. p4.

Morales, I.; Escalante, W. y Galdámez, I. (2015). Manejo agronómico del cultivo de Cilantro. FUNDESYRAM. El Salvador.

Ozuna, Gustavo. (2015). Fertilizantes órganominerales y ácido giberelico en el rendimiento y calidad del cilantro (Coriandrum sativum L.) Var. Marroquí. Trabajo de grado Ingeniero Agrónomo. Universidad Autónoma Agraria Antonio Narro. Saltillo, Coahuila, México. Pág. 50-53.

Palencia, G.; Mercado, T. y Combatt, E. (2006). Estudio agroclimático del departamento de Córdoba. Gráficas del caribe Ltda. Montería. 126p.

Rajeshwari, U. y Andallu, B. (2011). Medicinal benefits of coriander (Coriandrum sativum L.). Spatula DD 1(1): 51-58.
Ramadan, M.; Kroh, L. y Morsel, J. (2003). Radical scavenging activity of black cumin (Nigella sativa L.), coriander (Coriandrum sativum L.), and Niger (Guizotia abyssinica Cass.) crude seed oils and oil fractions. J. Agr. Food-Chem. 51: 6961-6969.

Salgado, I., Hernández, G., Suárez, Y., Mancera, M. y Guerra, D. (2020). Eficacia de métodos de desinfección y los efectos sobre las propiedades nutracéuticas en cilantro y fresa. Revista Mexicana de Ciencias Agrícolas, 11(2): 327-337.

Tituaña, E., Cayambe, J., Puerres, D., Heredia, M. (2019). Efectividad de sedimentos de la laguna de Colta como abono orgánico para la recuperación de suelos en el cultivo de cilantro. Revista Iberoamericana Ambiente \& Sustentabilidad, 2(3): $179-185$.

Torres N. (2012). Efecto de tres abonaduras orgánicas en el cultivo de apio (Apium graveolens) en la zona de la Libertad Cantón Espejo, Provincia del Carchi. Trabajo de grado Ingeniero Agrónomo. Universidad Técnica de Babahoyo Facultad de Ciencias Agropecuarias. Pág. 27.

Valdiviezo (2011). Identificación del agente causal de la mancha foliar denominada peca en culantro (Coriandrum sativum L.,) y establecer la eficiencia de control mediante productos orgánicos. Trabajo de grado Ingeniero Agrónomo. Escuela Superior Politécnica del Ejército. Ecuador. Pág. 2-4.

Vásquez, Alberto. (2006). Evaluación agronómica de once cultivares de Spinacia olerace L. para cultivo industrial en la zona de Valdivia. Trabajo de grado Ingeniero Agrónomo. Universidad Austral de Chile. Valdivia-Chile. Pág. 40. 